\title{
Practical estimation of sound reflection of a panel with a reflection coefficient
}

\author{
Yoshimasa Sakurai* and Koichi Nagata** \\ *Faculty of Engineering, Kansai University, \\ 3-3-35, Yamate-cho, Suita, Osaka, 564 Japan \\ **OTO Acoustic Engineering Associates, \\ 1-1-3, Kitahorie, Nishi-ku, Osaka, 550 Japan
}

(Received 13 August 1980)

\begin{abstract}
In an auditorium, an absorbent boundary is used in order to control the reverberant field. Its reflection composes a part of the early reflections and can not be neglected to estimate the transfer function. The sound reflection coefficient is defined as an unknown function of the integral equation of the first kind, referring to the reflection of a surface element on a rigid plane. It is very difficult with the equation to determine the coefficient which changes depending on incident and reflection angles. When a panel covered with a surface having a reflection coefficient which changes continuously and smoothly is large enough, the kernel function leaves the contribution around the specular reflection point and then the coefficient there can be separated experimentally. The reflection of a panel of limited dimension with such a reflection coefficient is approximately obtained by the convolution of the coefficient and the reflection of the rigid panel at the same position of the panel. Calculated and measured results are compared in the time and frequency domains. This method is also applied to a convex and a concave panel covered with a layer of such a reflection coefficient. The reflection of a panel with a mutual arrangement of strips of two different reflection coefficients is also discussed.
\end{abstract}

PACS number: $43.55 . \mathrm{Br}$

\section{DEFINITION OF THE SOUND REFLECTION COEFFICIENT AND THE APPLICATION TO A PANEL OF LIMITED DIMENSION COVERED WITH SUCH A SURFACE}

The reflection of a panel covered with an absorbent layer has been investigated in order to solve the velocity potential on the surface, ${ }^{1)}$ finding the relation between the velocity potential and its normal derivative for instance, with the assumption of the local reaction. To find a rigorous solution this approach is important, but not all surface materials necessarily satisfy the assumption and the calculation time needed increases extremely when the surface has a more complicated structure.

Without knowing the velocity potential and its normal derivative on the surface, the reflection diffusion function-tentatively named-is defined ${ }^{2)}$ by one of the authors as the coefficient which is referred to a rigid surface element and which explains the reflected wave at a receiving point. It is, in the result, equivalent to the reflection coefficient defined ${ }^{3)}$ for a spherical wave from the image of a point source, as is mentioned later. This definition is essentially the same as that of the local reflection coefficient which is defined in Ref. 4). The reflection coefficient is used in place of reflection diffusion function.

For a sufficiently large plane which is covered with a homogeneous layer and whose edge contribution is negligible, the sound reflection coefficient $R_{\omega}\left(\alpha_{i}\right.$, 


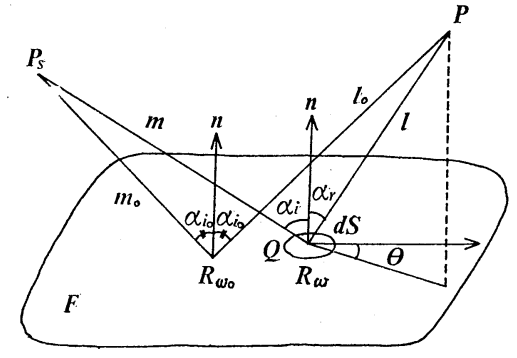

Fig. 1 Reflection of a sufficiently large plane panel covered with the surface of a reflection coefficient.

$\left.\alpha_{r}, \theta\right)$ referring to a rigid surface element is defined as follows, letting a velocity potential at a receiving point $P, \varphi_{\omega}(P)$ (see Fig. 1),

$$
\varphi_{\omega}(P)=\iint_{F} R_{\omega}\left(\alpha_{i}, \alpha_{r}, \theta\right) \cdot K_{\omega}\left(m, l, \alpha_{i}, \alpha_{r}\right) d F,
$$

where $K_{\omega}$ is an integrand for a rigid panel of the area $F$ and is discussed in Ref. 5), and $\alpha_{i}, \alpha_{r}$ and $\theta$ are incident, reflection and azimuth angles at a point $Q$ on the panel, respectively. It is the integral equation of the first kind as for an unknown function $R_{\omega}$ with the kernel $K_{w}$. When it is solved by a linear equation, the matrix of the coefficients makes it difficult, ${ }^{6)}$ and very precise measurements of $\varphi_{\omega}(P)$ in the left hand side are needed. A new approach is required to find the "local" reflection coefficient using the equation.

When the plane is large enough not to be affected by the boundary wave and $R_{\omega}$ changes smoothly and continuously, integration by Eq. (1) leaves only the contribution around the specular reflection point, because the integration of the kernel, which is multiplied by $R_{\omega}$, vanishes in the progress of the wavefront. Finally the reflection coefficient around there, $R_{\omega 0}$, is experimentally separated as in the following,

$$
\varphi_{\omega}(P)=R_{\omega 0} \cdot e^{-i k\left(m_{0}+l_{0}\right)} /\left(m_{0}+l_{0}\right),
$$

where $m_{0}$ and $l_{0}$ are the distances from a point source and a receiving point to the specular reflection point, and $k$ is a wave number.

The practical calculation for a panel of limited dimension covered with such a surface can be treated as follows.

When a point source and a receiving point are fixed, $R_{\omega}\left(\alpha_{i}, \alpha_{r}, \theta\right)$ is said to depend also on the coordinates of the plane $(x, y) . \quad R_{\omega}(x, y)$, which is separate from the specular reflection point $(a, b)$ by $\mathrm{h}$ to $x$ direction and $\mathrm{k}$ to $y$ 's, is developed into the Taylor series,

$$
\begin{aligned}
& R_{\omega}(x, y)=R_{\omega}(a+\mathrm{h}, b+\mathrm{k}) \\
& \quad=R_{\omega}(a, b)+\left\{\partial R_{\omega} / \partial x \cdot \mathrm{h}+\partial R_{\omega} / \partial y \cdot \mathrm{k}\right\} / 1 !+\cdots,
\end{aligned}
$$

where $\partial R_{\omega} / \partial x$ is a partial derivative to the $x$ direction and $\partial R_{\omega} / \partial y$ is one to $y$ 's at $(a, b)$. When $\mathrm{h}$ and $\mathrm{k}$ are small and/or $\partial R_{\omega} / \partial x$ and $\partial R_{\omega} / \partial y$ are small, $R_{\omega}(x, y)$ can be replaced by $R_{\omega}(a, b)$ namely $R_{\omega 0}$.

The behavior of the function $K_{\omega}$, being multiplied to a smoothly changing $R_{\omega}$ and being integrated, leaves little effect till the wave reaches the boundary after the specular reflection point. When $h$ and $k$ at the edge are larger, the boundary wave becomes smaller. ${ }^{5)}$ Even if a reflection coefficient there varies from $R_{\omega 0}$, then its convolution with the boundary wave of a rigid panel does not affect that of the geometrical wave.

Finally the reflection of a plane panel of area $F$ covered with a surface having a reflection coefficient is approximately given ${ }^{2)}$ by,

$$
\varphi_{\omega}(P)=R_{\omega 0} \iint_{F} K_{\omega} d F=R_{\omega 0} \cdot H_{\omega}(P),
$$

where $H_{\omega}(P)=\iint_{F} K_{\omega} d F$ and is the reflection of the rigid plane panel which is in the same place of the panel. $R_{\omega 0}$ must be measured in advance.

$\phi(P, t), r_{0}(t)$, and $h(P, t)$ are the inverse Fourier transforms of $\varphi_{\omega}(P), R_{\omega 0}\left(\alpha_{i}\right)$, and $H_{\omega}(P)$, respectively, being related by,

$$
\begin{aligned}
\phi(P, t) & =\int_{-\infty}^{\infty} \varphi_{\omega}(P) e^{i \omega t} d \omega, \\
r_{0}(t) & =\int_{-\infty}^{\infty} R_{\omega 0}\left(\alpha_{i}\right) e^{i \omega t} d \omega,
\end{aligned}
$$

and

$$
h(P, t)=\int_{-\infty}^{\infty} H_{\omega}(P) e^{i \omega t} d \omega .
$$

Equations (2) and (4) are expressed as follows,

$$
\begin{gathered}
\phi(P, t)=r_{0}\left\{t-\left(m_{0}+l_{0}\right) / C\right\} /\left(m_{0}+l_{0}\right) \\
\phi(P, t)=r_{0}(t) * h(P, t)
\end{gathered}
$$




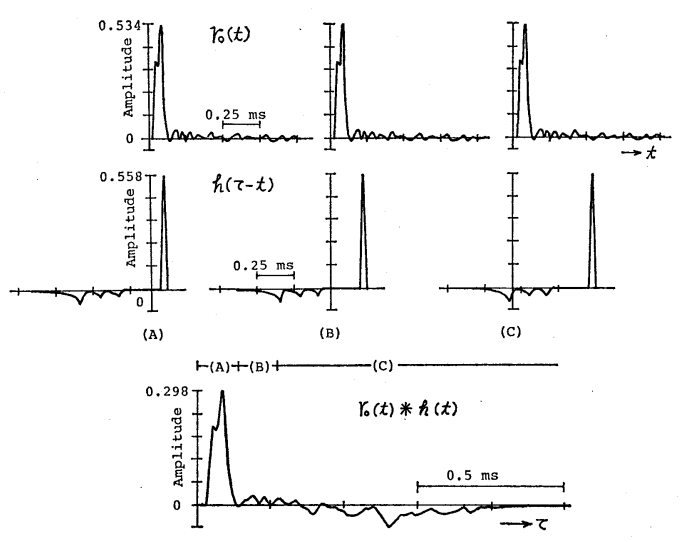

Fig. 2 Convolution of a reflection coefficient and the impulse response of a rigid plane panel.

It should be noticed that $r_{0}(t), h(\tau-t)$ and their convolution are made smooth by the filter. ${ }^{5}$ )

where $*$ shows the convolution product.

The convolution product in Eq. (9) is illustrated in Fig. $2{ }^{7)}$ where a panel has the specular reflection point on it. Integration of the product $r_{0}(t)$ and $h(\tau$ $-t$ ) in (A), (B) and (C) is in the range (A), (B) and (C) of the figure in the bottom, respectively. It is convenient to see the validity of the treatment in Eqs. (4) and (9).

$r_{0}(t)$ is easily obtained from Eq. (8) by the acoustical measurement of $\phi(P, t)$ and the lengths of $m_{0}$ and $l_{0}$. Recording in the form of $r_{0}(t)$ gives easily the reflection coefficient in the frequency domain by the fast Fourier transform technique.

There are many kinds of porous layers whose reflection coefficients are not changed by varying the distances $m_{0}$ and $l_{0}$ as shown later. It can be imagined that the reflection coefficient for an uneven surface or the surface with a thick layer might not be able to regard as constant, but Eq. (9) is still the approximation as long as a point source and a receiving point for a panel are in the same locations as those for measuring the reflection coefficient by a large plane. When a panel is small and a surface layer is thick, its edge has quite different condition as that of a large plane, and the secondary disturbance there can not be explained by this method.

\section{MEASUREMENT OF REFLECTION COEFFICIENT FOR A LARGE PLANE}

The reflection coefficient for a spherical wave incidence, in the frequency domain, is measured by the interference pattern method ${ }^{3)}$ and the impulse method $^{8)}$ by a sufficiently large plane covered with an absorbent porous layer. Pulse response of a porous layer with a rigid back is measured for the plane wave incidence. ${ }^{9)}$ The reflection coefficients in the frequency domain $R_{\omega 0}$ and in the time domain $r_{0}(t)$ for a sufficiently large plane covered with the surface of the reflection coefficient are measured $^{6)}$ by an impulsive spherical wave incidence.

Porous layers of punch-carpet (about $4 \mathrm{~mm}$ thick) and urethane foam $(25 \mathrm{~mm}$ thick) are used for covering plane panels which are plywoods of $180 \mathrm{~cm}$ $\times 180 \mathrm{~cm}$ with a thickness of $9 \mathrm{~mm}$. Measured waves are digitally processed ${ }^{5)}$ with FFT technique. Reflection coefficient in the time domain is inversely Fourier transformed from the filtered reflection coefficient in the frequency domain.

Reflection coefficients $r_{0}(t)$ and $R_{\omega 0}$ at every 20 degrees from 0 to 80 degrees are shown in Figs. 3 and 4, respectively. Reflection coefficient in the frequency domain $R_{\omega 0}$ is expressed in the form of $20 \log _{10}\left|R_{\omega 0}\right|$. Zero decibel of the ordinate in the frequency domain corresponds to the reflection coefficient of the rigid surface, which is unity.

The duration of the spark pulse is about $0.7 \mathrm{~ms}$. It was easy to separate the direct wave and a reflected wave till the incident angle was $60^{\circ}$. At the incident angle of $80^{\circ}$, a wave was recorded by two microphones at the same time and then they were separated. The locations of the point source and a receiving point for the measurements are listed in Table 1 whose coordinates are in the figure.

The first peak in the time domain is reflected on the surface and the second is reflected by the rigid back plane. The punch-carpet plane gives more

Table 1 Geometry of a point source and a receiving point for measuring a reflection coefficient in $\mathrm{cm}$.
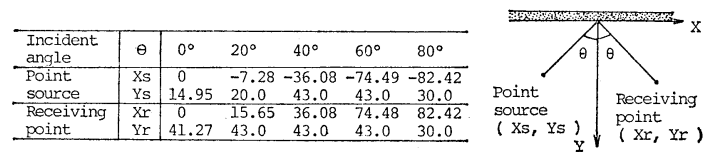


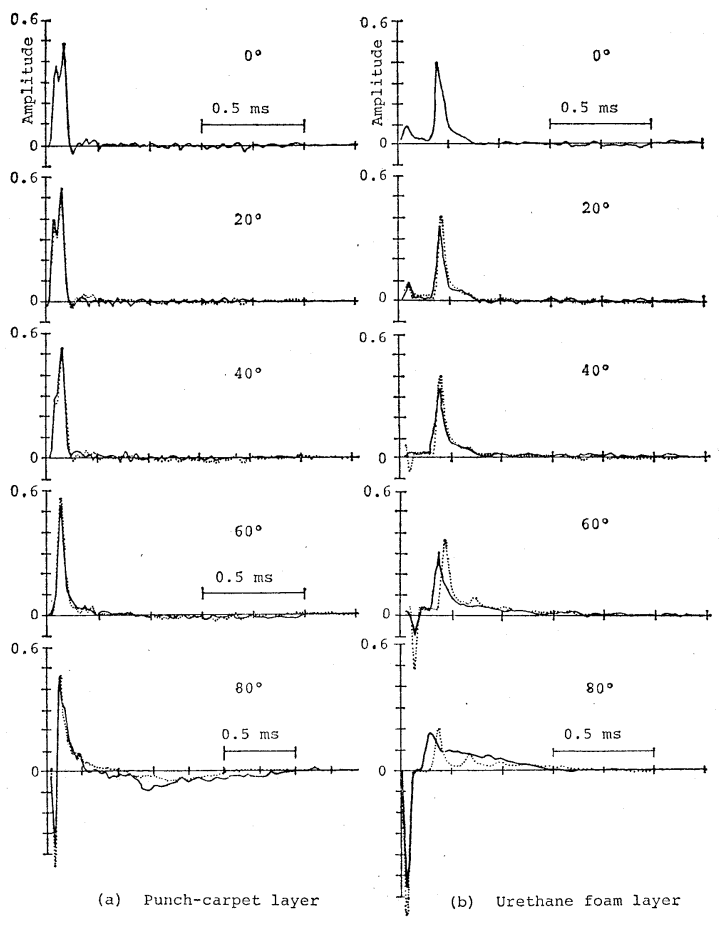

Fig. 3 Reflection coefficients, in the time domain, of the surfaces of (a) punchcarpet and (b) urethane foam layers at the incident angles 0 to $80^{\circ}$.

Dotted lines are calculated by the assumption of local reaction.

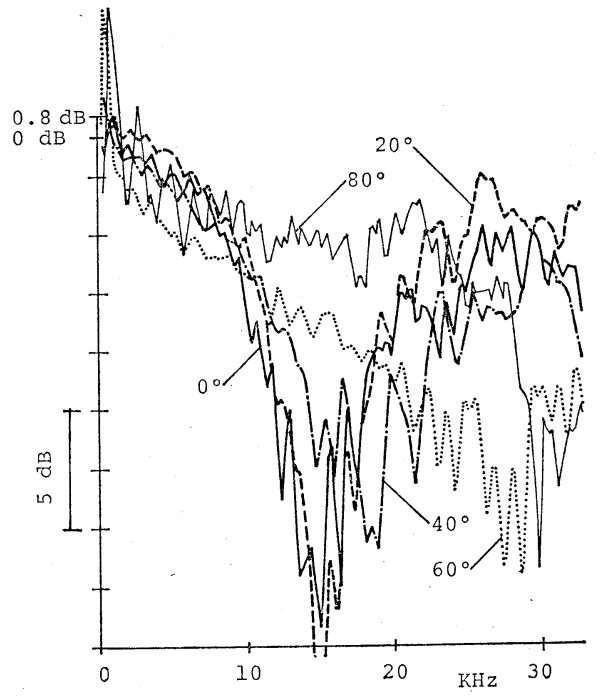

(a) Punch-carpet layer surface reflection because of the larger impedance mismatching on it. Thus the reflection coefficient in the time domain is directly physically expressed. It is furthermore interesting that at the grazing angle the surfaces of both materials reflect the surface reflection in the negative side.

The reflection coefficient in the frequency domain changes depending on an incident angle at the specular reflection point.

When an admittance is obtained from the reflection coefficient at the normal incidence, and the local reaction for a plane wave incidence is assumed, the reflection coefficient in the time domain at a different incident angle is calculated by the inverse Fourier transform of that in the frequency domain and is plotted with a dotted line in Fig. 3. The reflection coefficient by the assumption of the local reaction also yields the negative first peak in the grazing angle. As an incident angle approaches the grazing angle, they tend to differ each other especially for a urethane foam layer. Further investigation of the sphericity of wave front and extended reaction ${ }^{10)}$ seems to be needed.

When the locations of a point source and a receiving point are different as shown in Table 2 at the incident angle of 40 degrees, the change of reflection coefficients are shown in Fig. 5 for a punch-carpet surface and in Fig. 6 for a urethane foam surface.

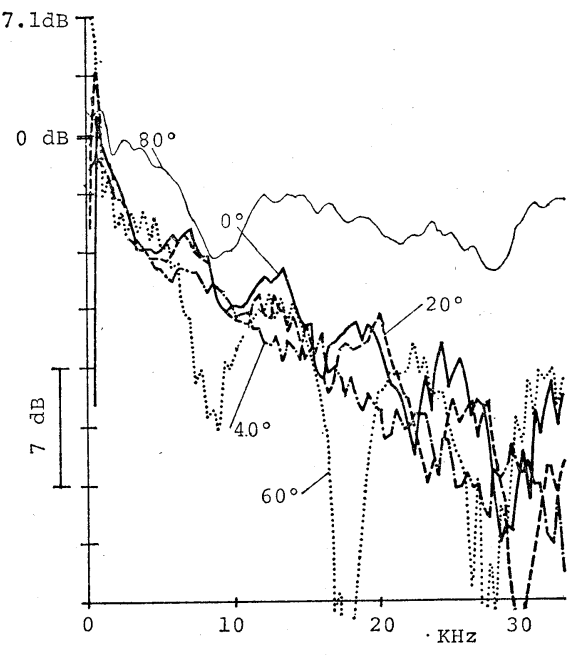

(b) Urethane foam layer

Fig. 4 Reflection coefficients, in the frequency domain, of the surfaces of (a) punch-carpet and (b) urethane foam layers. 


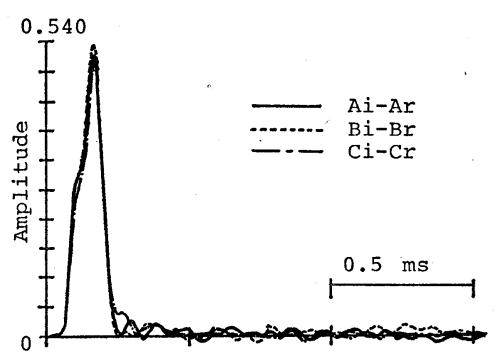

(a) Time domain

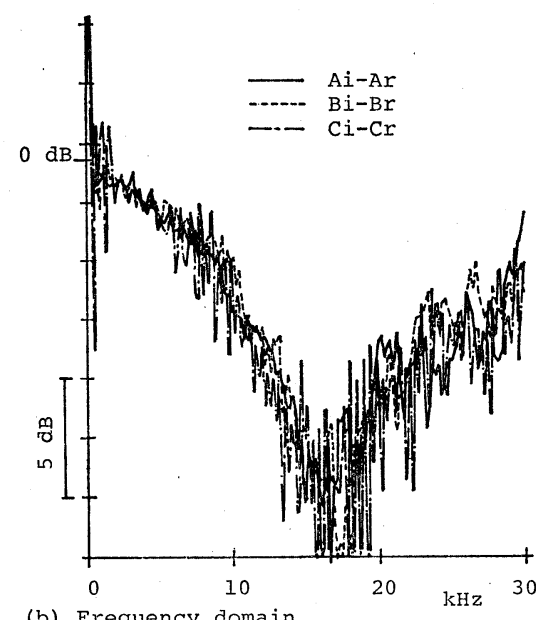

(b) Frequency domain

Fig. 5 Change of reflection coefficients of a punch-carpet layer by different locations of a point source and a receiving point in Table 2.

Sampling points for $\mathrm{Ai}-\mathrm{Ar}$ and $\mathrm{Bi}-\mathrm{Br}$ are 1024 , and those for $\mathrm{Ci}-\mathrm{Cr}$ are 2048 with the time interval of $5 \mu$ s. Great difference among them is not observed in these cases.

\section{PANEL OF LIMITED DIMENSION COVERED WITH \\ A HOMOGENEOUS LAYER}

\subsection{Plane Panel ${ }^{6)}$}

In this paper, calculated and measured impulse responses are inversely Fourier transformed from their filtered ${ }^{5,11)}$ transfer functions. Zero decibel in the ordinate of a transfer function refers to the direct sound pressure level from the image of a point source for a plane panel or that from a point source for a curved panel. Measured values are shown by a solid line and calculated ones by a dotted line. Transfer function of a rigid panel used

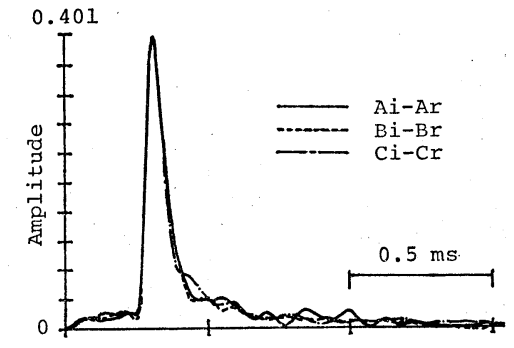

(a) Time domain

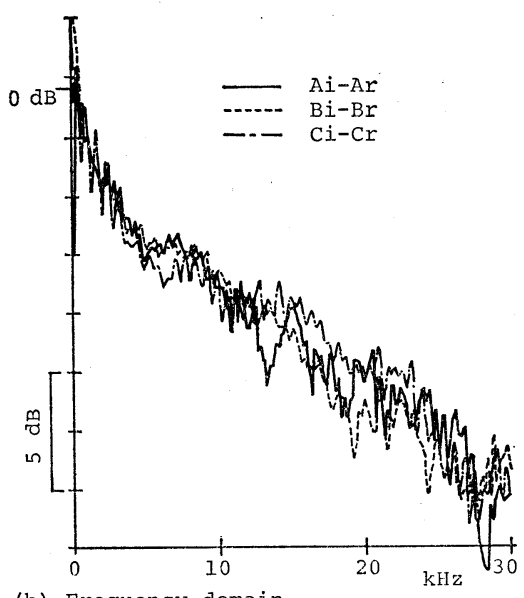

(b) Frequency domain

Fig. 6 Change of reflection coefficients of a urethane foam layer by different locations of a point source and a receiving point in Table 2.

Table 2 Different locations of a point source and a receiving point for measuring a reflection coefficient at the incident angle 40 degrees in $\mathrm{cm}$.

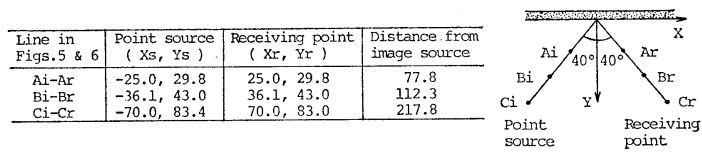

for convolution is also shown by a dashed line.

A panel in Fig. 7 is covered with a porous layer. Both panels have specular reflection points on them. $h(P, t)$ for a rigid panel is estimated by the line integral. ${ }^{5)}$ Transfer function and impulse response calculated by Eqs. (4) and (9) are compared with measured values in Figs. 8 and 9 for the panels covered with punch-carpet in Fig. 7 (a) and urethane foam in Fig. 7 (b), respectively. 


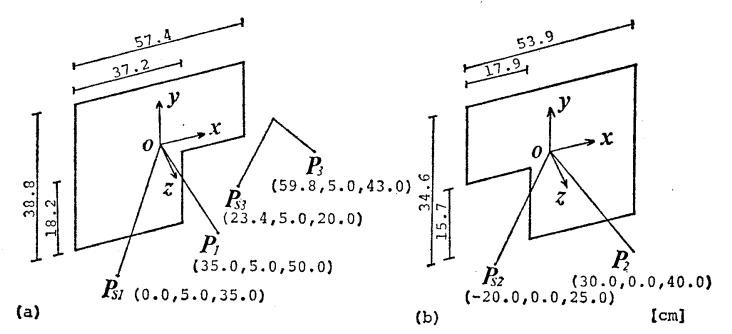

Fig. 7 A plane panel covered with the surface of a reflection coefficient.

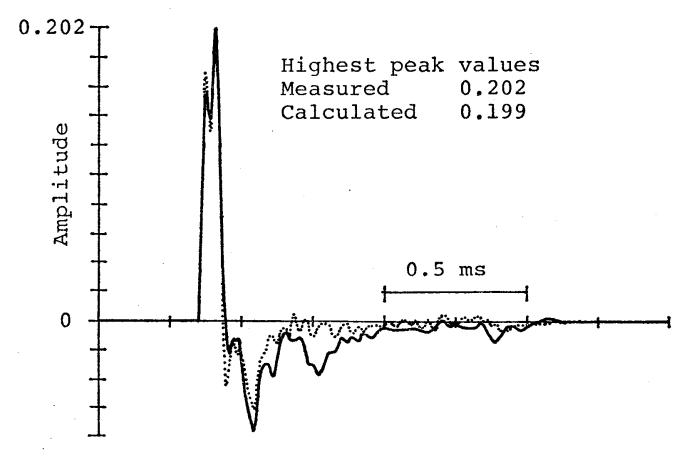

(a) Impulse Response

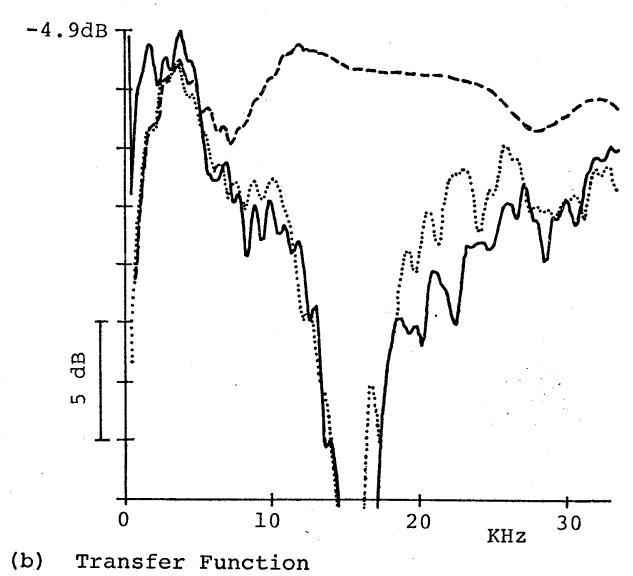

Fig. 8 Reflection of a plane panel of limited dimension in Fig. 7(a) covered with a punch-carpet layer.

A point source and a receiving point are at $P_{S 1}$ and $P_{1}$, respectively.

Though a little difference is observed for the boundary wave of both panels which is considered to be caused by the diffraction into the back of a panel being neglected in the calculation of $h(P, t)$,

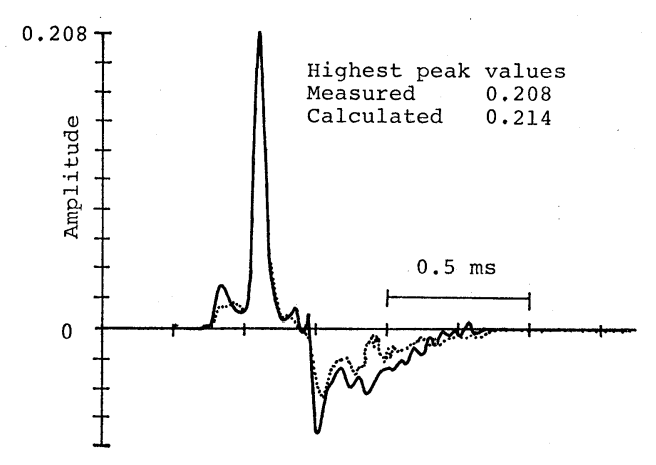

(a) Impulse Response

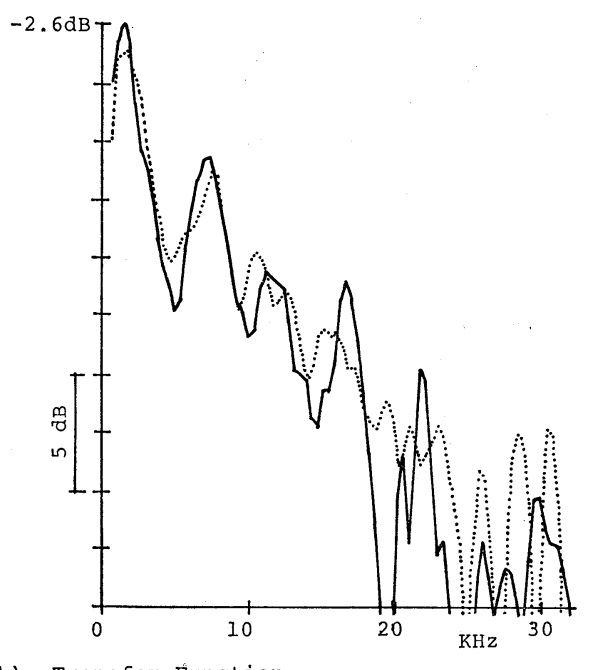

(b) Transfer Function

Fig. 9 Reflection of a plane panel in Fig. 7(b) covered with a urethane foam layer. A point source is at $\boldsymbol{P}_{S 2}$ and a receiving point at $P_{2}$.

and the secondary disturbance at the edge, on the whole they correspond with each other.

When a point source and a receiving point are at $P_{S 3}$ and $P_{3}$ in Fig. 7, respectively, and the specular reflection point is out of the panel, the reflection coefficient at the incident angle to the point is convoluted with $h(P, t)$ of the rigid panel whose surface is at the same place as that of the panel. Calculated values shown by a dotted line and measured ones by a solid line are compared in Fig. 10 for the panel covered with punch-carpet. Though the signal to noise ratio is not sufficient, it is observed that the highest peaks are close to each other. The reflection without the specular reflection point is 


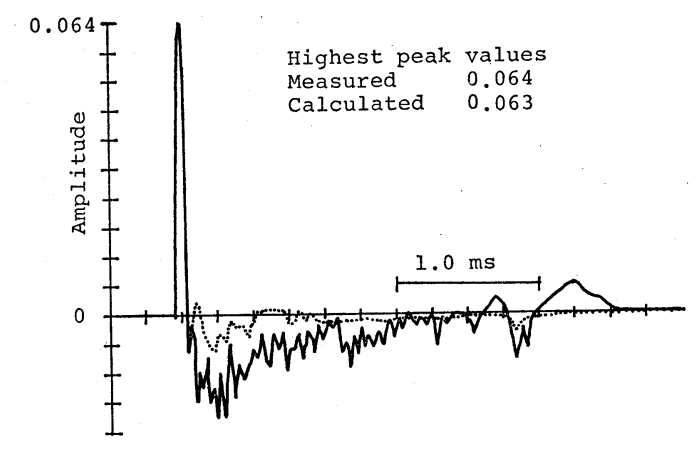

(a) Impulse Response

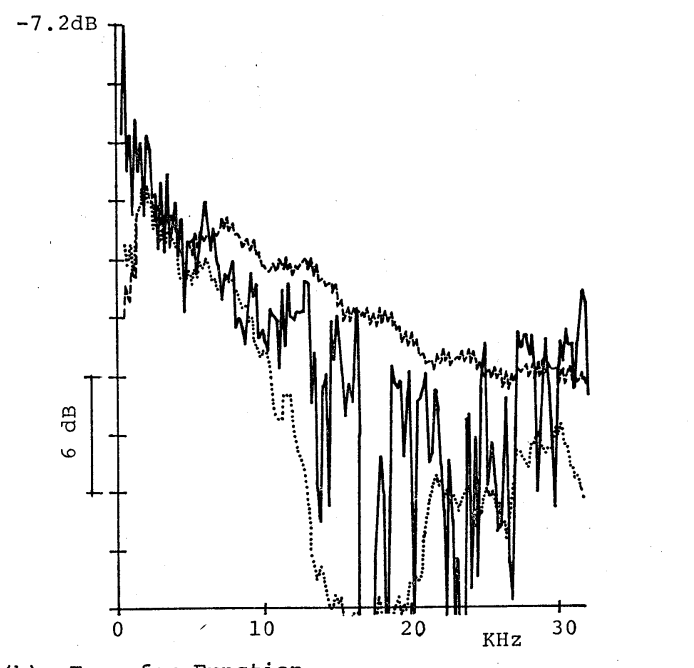

(b) Transfer Function

Fig. 10 Reflection of a plane panel covered with a punch-carpet layer in Fig. 7(a) which has no specular reflection point on it.

A point source is at $\boldsymbol{P}_{S 3}$ and a receiving point is at $\boldsymbol{P}_{3}$.

much smaller than that with the point, because the reflection of the rigid plane panel itself is small when it has no point on it. It seems that the reflection coefficient on the panel is not greatly different from that at the specular reflection point in this case.

\subsection{Convex and Concave Panels}

The impulse response of a convex or a concave rigid panel has a discrete peak $^{11)}$ caused by the contribution around the specular reflection point and resembles that of a rigid plane panel. It suggests that the reflection of a curved panel covered with

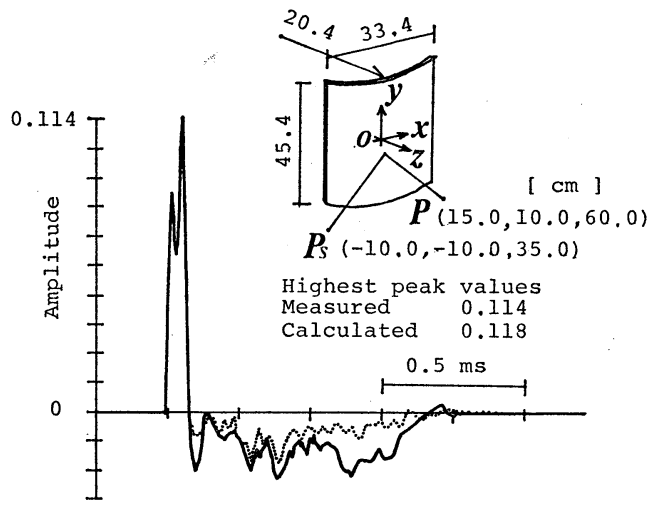

(a) Impulse Response

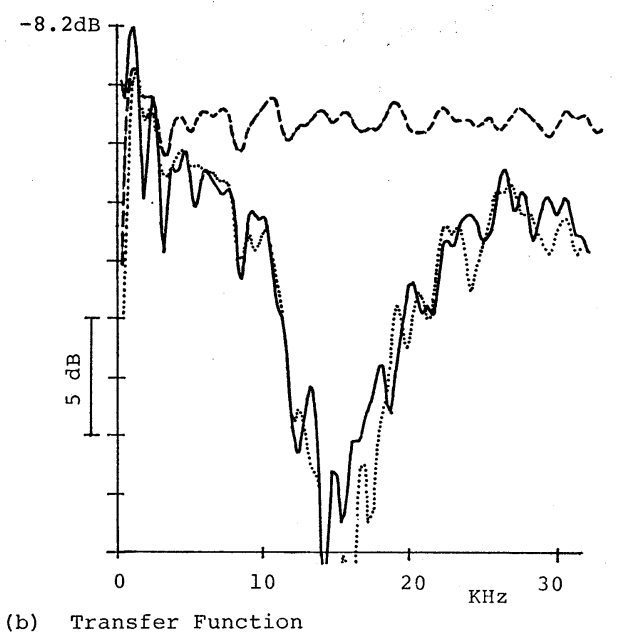

Fig. 11 Reflection of a convex panel covered with a punch-carpet layer.

a homogeneous layer is predictable by Eq. (9), i.e., by the convolution of the reflection coefficient at the specular reflection point on it and the reflection of the rigid curved panel whose surface is at the same place as that of the panel, though a curved surface has gradually changing incident and reflection angles, and different reflection coefficients. The calculation of $h(P, t)$ for a curved rigid panel is reported in Ref. 11).

Calculated and measured reflections of convex and concave panels covered with punch-carpet are compared in Figs. 11 and 12, respectively. A solid line shows the measured result and a dotted line is calculated by the convolution of Eq. (9) when the impulse response of a rigid curved panel is expressed 


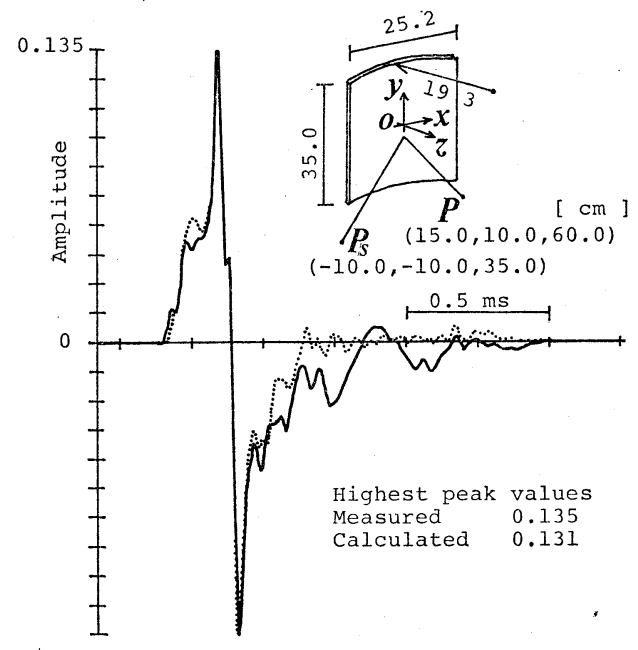

(a) Impulse Response

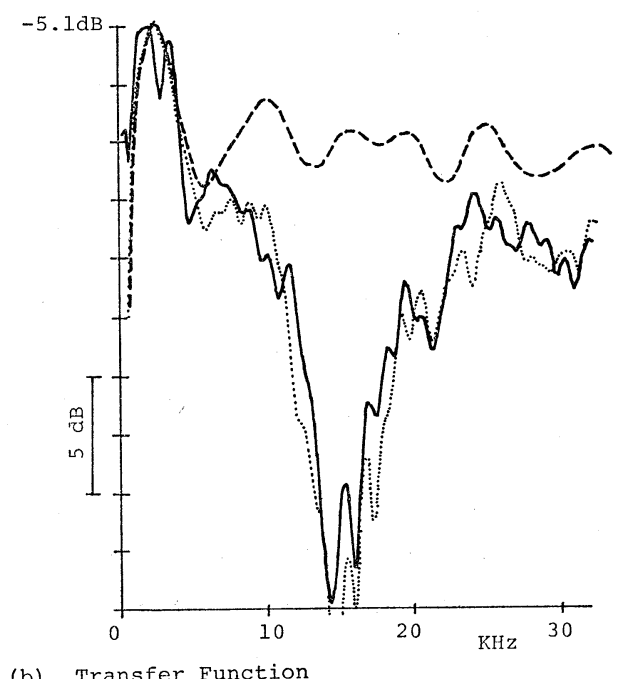

(b) Transfer Function

Fig. 12 Reflection of a concave panel covered with a punch-carpet layer.

as a dashed line. Reflections are normalized by the direct sound from a point source. A small difference is observed in the later parts of the boundary waves. It is supposed to be caused by the edge effects and the different multiplex reflection in the curved layer.

In Fig. 13, where the lines show the same results as in Figs. 11 and 12, the concave panel has a second discrete reflection from the other part of its inside. Evident difference is recognized at more than about $16 \mathrm{kHz}$. It must be caused by the reflection coefficient at the different incident angle for the

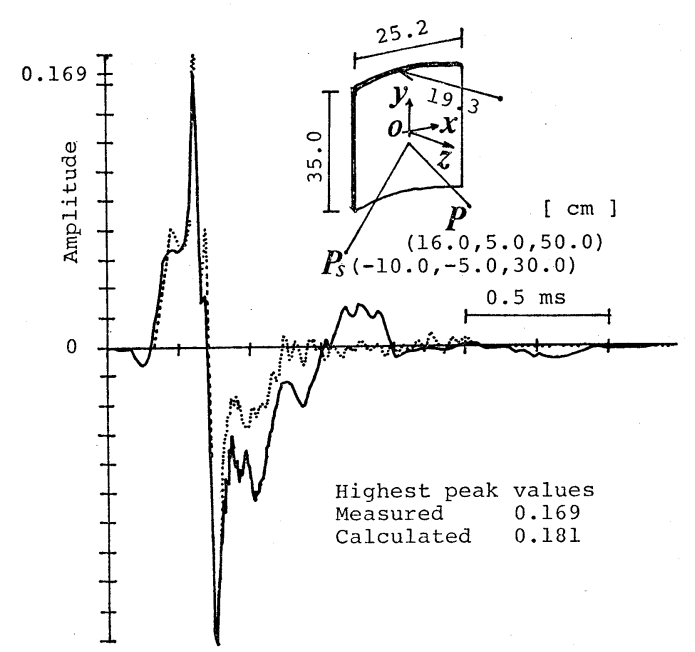

(a) Impulse Response

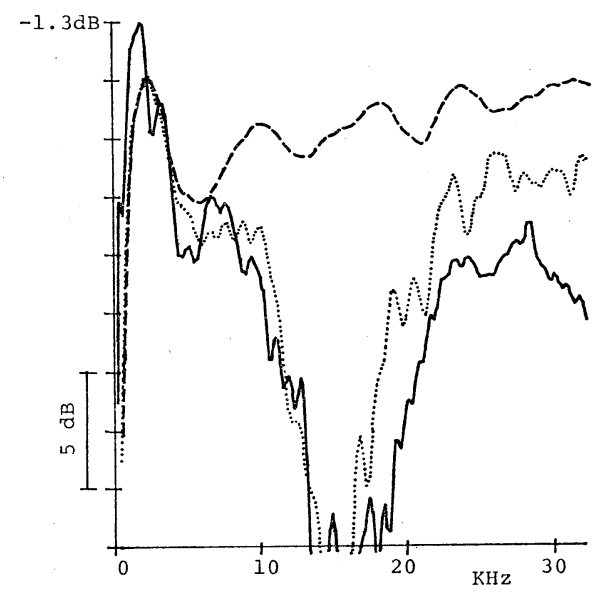

(b) Transfer Function

Fig. 13 Reflection of a concave panel covered with a punch-carpet layer, having a discrete second reflection from the other part of its inside.

second reflection.

\section{MUTUAL ARRANGEMENT OF RECTANGULAR STRIPS COVERED WITH MATERIAL OF TWO DIFFERENT KINDS OF REFLECTION COEFFICIENTS ${ }^{12)}$}

4.1 Punch-Carpet and Rigid Surface

Rectangular strips covered with rigid plane and punch-carpet layer are mutually arranged at $\mathrm{A}$ and B, as in Fig. 14, respectively. The punch-carpet 


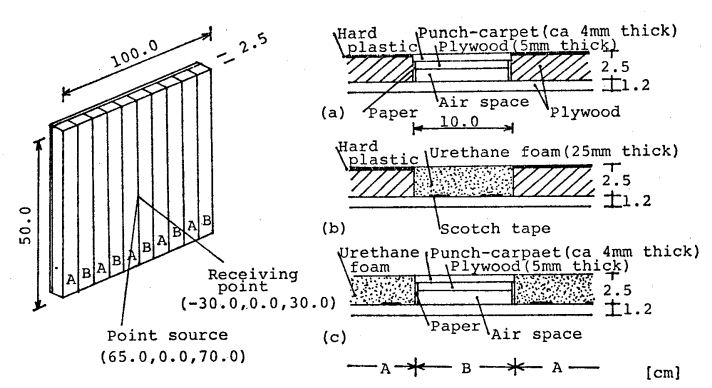

Fig. 14 Mutual arrangement of strips covered with two different kinds of surfaces. A point source and a receiving point are at the same positions as shown in figure for three panels (a), (b) and (c).

layer covers a plywood strip of $6 \mathrm{~mm}$ thick and its back space is surrounded by a thick sheet of paper having a depth of $14 \mathrm{~mm}$, as shown in Fig. 14(a). The punch-carpet used here and in Fig. 17 is a different product from one whose reflection coefficients are shown in Fig. 3.

The reflection of the panel in Fig. 14 is calculated by the superposition of that of each strip. The reflection of a strip which is covered with punchcarpet and has no specular reflection point on it is replaced by the convolution of its reflection coefficient at the incident angle of the specular reflection on the panel and the reflection of the rigid rectangular strip at the same place as that of the strip.

Calculated and measured results are compared in Fig. 15 by a dotted line and a solid one, respectively. They agree well, though a little difference at 13 to $20 \mathrm{kHz}$ is observed.

\subsection{Urethane Foam and Rigid Surface}

Urethane foam of $25 \mathrm{~mm}$ thick is replaced at B as in Fig. 14 (b). The calculation is done in the same way as in 4.1. Measured and calculated results are compared in Fig. 16 by solid and dotted lines, respectively. The measured reflection is a little smaller than the calculated one at more than around $17 \mathrm{kHz}$. The urethane foam layer is relatively thick and the excess attenuation is caused by the multiplex reflection at the boundary in the layer. The contribution of the rigid strips is predominant and then impulse response and transfer function

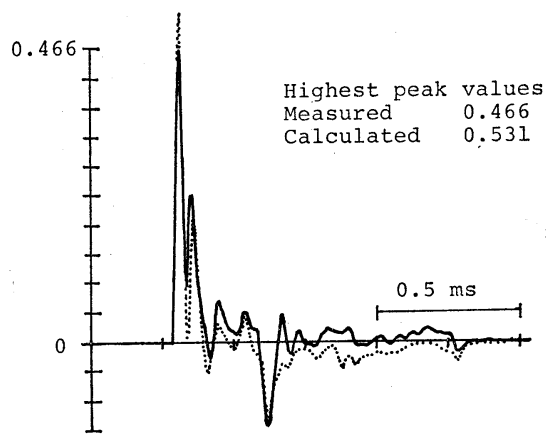

(a) Impulse Response

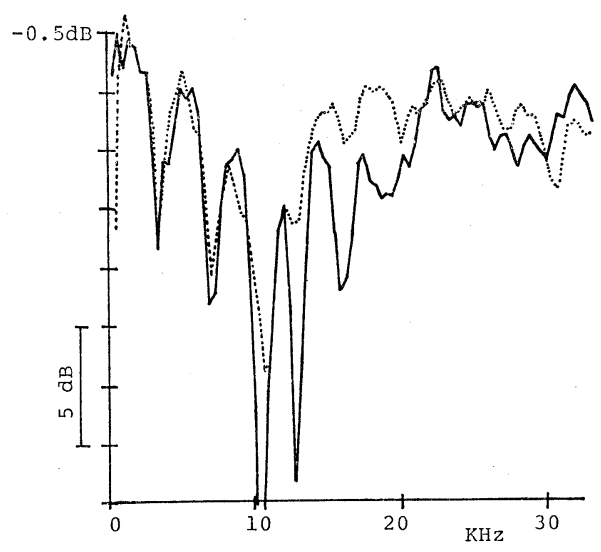

(b) Transfer Function

Fig. 15 Reflection of a plane panel covered with punch-carpet and rigid strips shown in Fig. 14(a).

are still in good agreements as in 4.1.

\subsection{Punch-Carpet and Urethane Foam}

4.3.1 Superposition of the reflection of each strip

The rectangular strips of punch-carpet and urethane foam whose reflection coefficients are less than unity are mutually arranged as in Fig. 14(c). The reflection of the panel is calculated by the superposition of the reflection of each strip as in 4.1 and 4.2, and is shown by a dotted line in Fig.17. Measured values shown by a solid line are smaller than calculated ones at more than around $20 \mathrm{kHz}$. The first peaks in the time domain correspond each other, but the later peaks do not. If the layer becomes thick as in this case, the multiplex reflection emerges at the boundary between two neighboring strips and it can not be explained by the superposition of the reflection of each surface. 


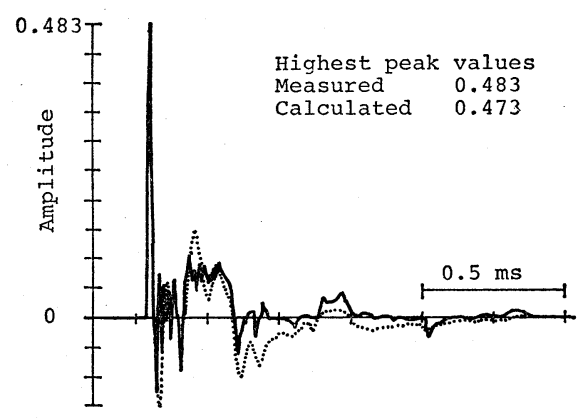

(a) Impulse Response

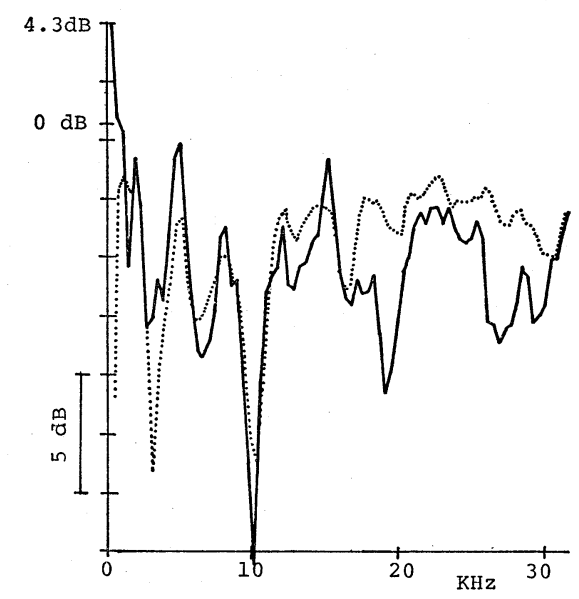

(b) Transfer Function

Fig. 16 Reflection of a plane panel covered with urethane foam and rigid strips shown in Fig. 14(b).

\subsubsection{Convolution with the reflection coefficient} obtained for a large plane panel of this kind Making the panel in Fig. 14 (c) sufficiently large in the same arrangement, a specimen of $180 \mathrm{~cm} \times$ $180 \mathrm{~cm}$ for measuring the reflection coefficient in Eq. (8) is obtained. The coefficients at the incident angles of 0,30 and 60 degrees are measured and shown in Fig. 18. Though it is an unhomogeneous surface, the reflection coefficient in the time domain rapidly decreases. The deviation in the time domain is thought to be caused by the mutual change of the surface materials.

The reflection coefficient calculated by the assumption of local reaction as in $\mathbf{2}$ is shown by a dotted line in Fig. 18 (a).

The reflected wave of the panel which has the specular reflection point and whose point source and receiving point are at the same positions as the

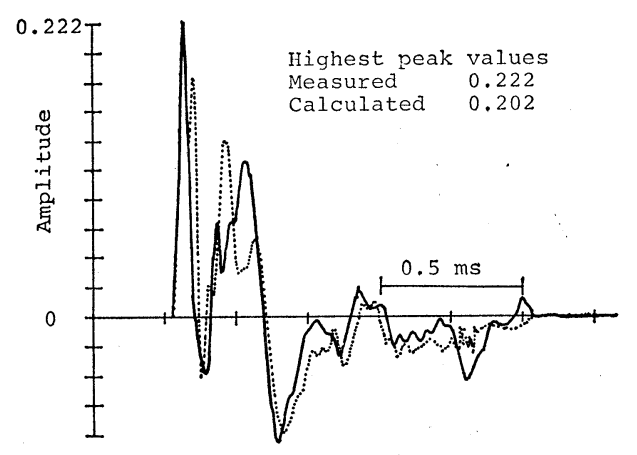

(a) Impulse Response

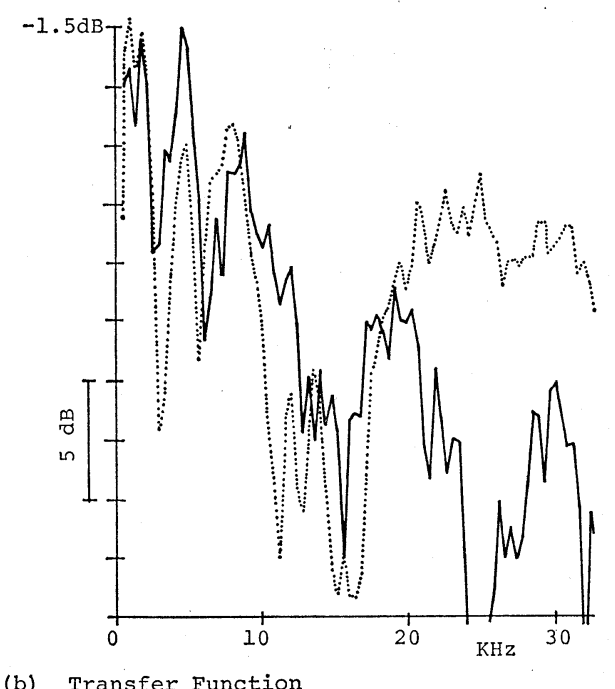

Fig. 17 Reflection of a plane panel covered with punch-carpet and urethane foam strips shown in Fig. 14(c).

coefficient at 30 degrees is measured, is calculated by the convolution of the reflection coefficient and the reflection of the rigid panel replaced at the panel in Fig. 19. The calculated and measured results, which are shown by dotted and solid lines, respectively, are compared in Fig. 20 in good agreement except for a slight difference in the later time domain. The same calculation is also applied to the panel and compared with the measured value in Fig. 21, when a receiving point is moved to $P_{2}$. In spite of a different receiving point the calculated values correspond well to the measured ones.

When a point source and a receiving point are at $P_{S 2}$ and $P_{3}$ in Fig. 19, respectively, and the panel has no specular reflection point, the same calcula- 


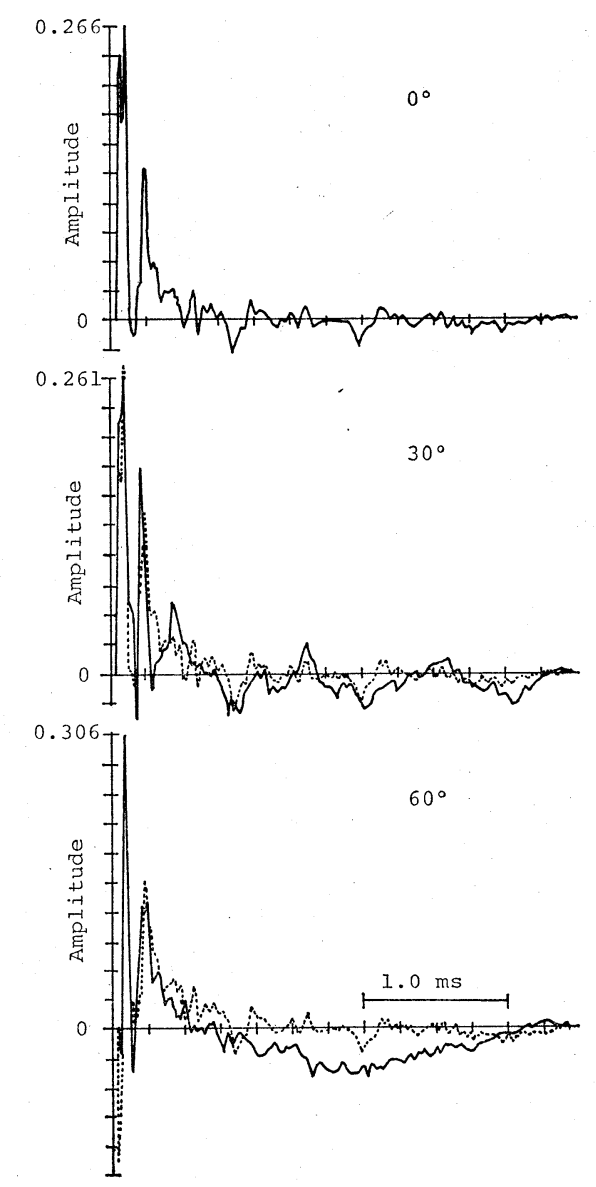

(a) Time Domain tion as mentioned in 3.1 is applied to it and its values are shown in Fig. 22 by a dotted line as the first calculation, which is different from measured values shown by a solid line. The reflection coefficient of the panel must be quite different from that obtained at the specular reflection point on the large plane.

Because the reflection coefficients of punchcarpet and urethane foam seem not to vary so much from those at the specular reflection point, and the multiplex reflection in the urethane foam layer seems not to be caused evidently as in the case of Fig. 17 in this geometrical relation, the reflection of the panel is calculated by the superposition of the reflection of each strip which is estimated by the convolution as in 4.1 and 4.2. The result is shown by a dashed line as the second calculation, and explains the measured values better in this case.

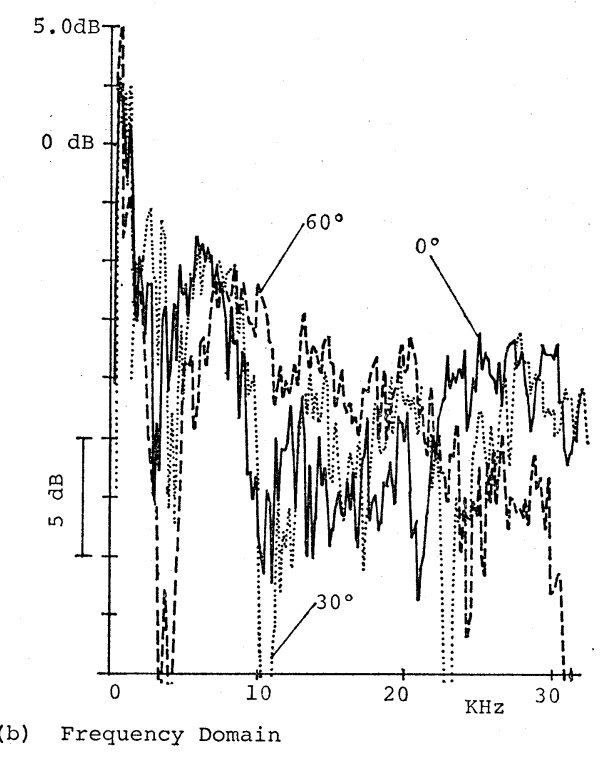

Fig. 18 Reflection coefficients for a large plane surface covered mutually with two different kinds of strips.

(a) In the time domain and (b) in the frequency.

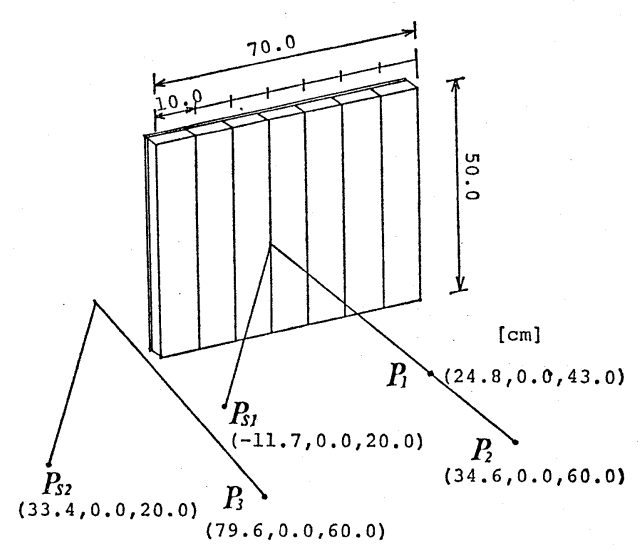

Fig. 19 A rectangular plane panel obtained from a large panel by which reflection coefficient in Fig. 18 is measured. 


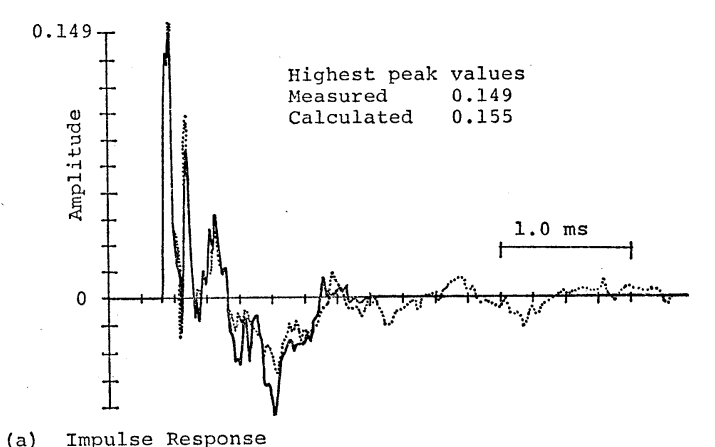

(a) Impulse Response

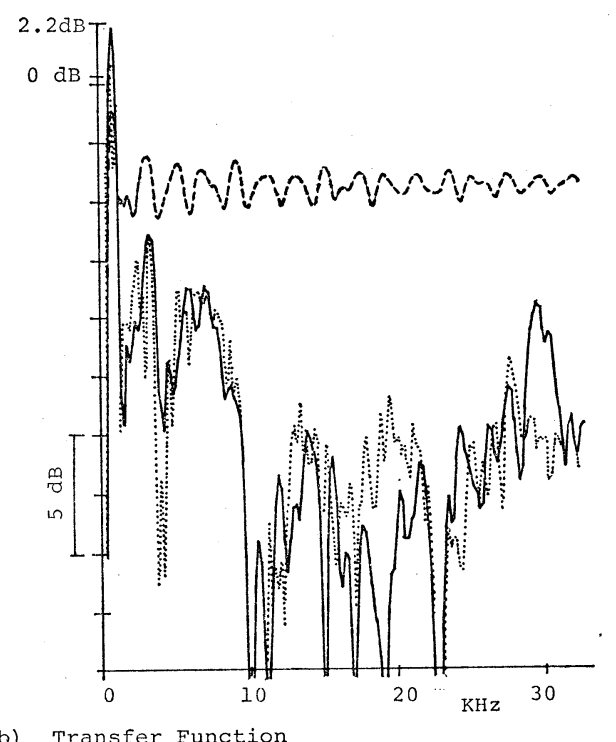

Fig. 20 Reflection of a plane panel shown in Fig. 19 at a receiving point $P_{1}$.

\section{CONCLUSION}

The reflection of a plane panel which is covered with a layer of a homogeneous material and which has a specular reflection point can be predicted by the convolution of the sound reflection coefficient at the incident angle to the point for a sufficiently large plane with the layer and the reflection of the rigid plane panel at the same position as the panel. Even if the specular reflection point is out of the panel and as long as the reflection coefficient is not varied there, the same convolution compares with the experiment very well.

The reflected wave of a convex or a concave panel covered with such material is also approximated by

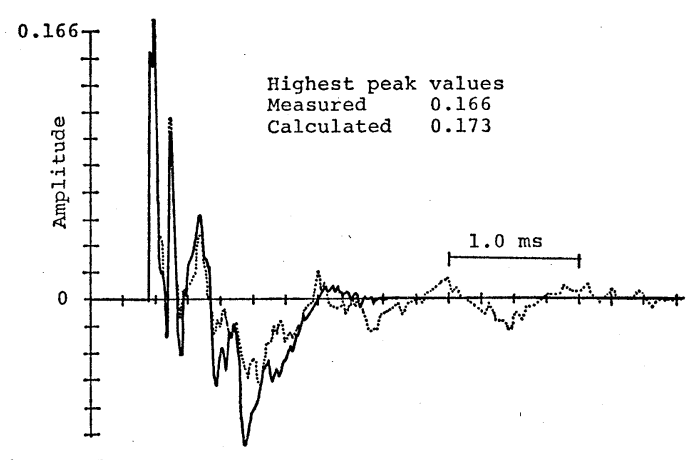

(a) Impulse Response

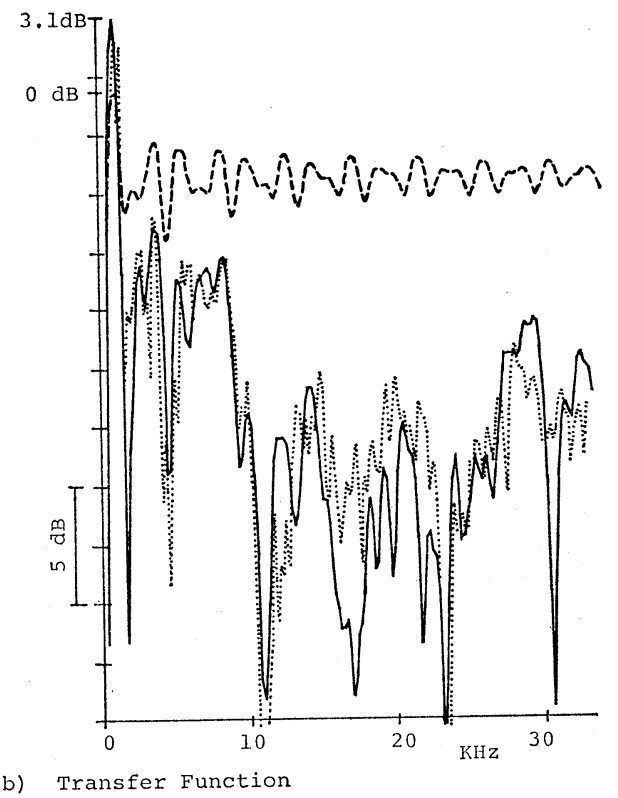

Fig. 21 Reflection of a plane panel shown in Fig. 19 at a receiving point $P_{2}$.

the convolution of the reflection coefficient at the incident angle of the specular reflection point on it and the reflection of such a rigid curved panel. When a concave panel has a discrete second reflection from the other part of its inside, a reflection coefficient must be taken at its incident angle and be convoluted by the second reflection of the rigid concave panel.

When a plane surface is covered with an unhomogeneous layer, and the reflection of the rigid parts is predominant or the absorbent layer is very thin compared with an incident wavelength, the reflection of such a panel is predictable by the 


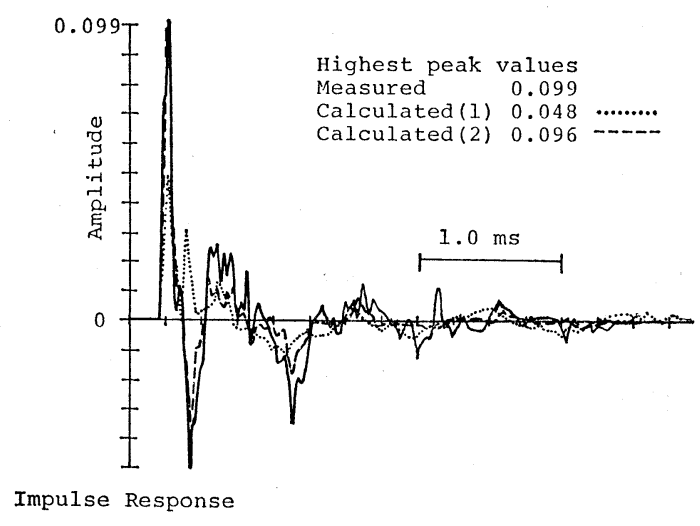

Fig. 22 Reflection of a plane panel shown in Fig. 19 at a receiving point $P_{3}$ with no specular reflection point on it.

A point source is at $\boldsymbol{P}_{S 3}$.

superposition of the reflection from each part that is obtained by the convolution of reflection coefficient and the reflection of the rigid plane of each part.

When a layer is relatively thick and the reflection coefficients at the other parts are less than unity, the excess attenuation by the multiplex reflection at the layer's boundary emerges and the superposition of the reflection of each part can not explain the measurement. Even if a surface is complicated as in this case, when the reflection coefficient is obtained from a sufficiently large plane panel of this structure and when a panel has the specular reflection point on it, the convolution of the reflection coefficient and the reflection of the rigid plane panel at the same place of the panel predicts the measured value sufficiently, at least at the same position as the reflection coefficient is measured.

More investigation concerning the reflection of a panel which does not have the specular reflection point on it might be needed, though the reflection itself is very small and is considered not greatly to affect a transfer function of an auditorium.

Though this method is not suitable for predicting the rigorous reflection including the effects of the diffraction into the back of a panel and the secondary disturbance at the edge, it is a very practical way of estimating the early reflections of this kind in an auditorium without finding the velocity potential and its normal derivative on the surface.

\section{ACKNOWLEDGEMENTS}

The authors wish to express their appreciation to Dr. T. Terai, Professor at Kyoto Univ., and Mr. Y. Kawai, teaching and research assistant at Osaka Institute of Technology for their participation in useful discussions. They also wish to express thanks to Dr. Z. Maekawa, Professor at Kobe Univ., and Dr. K. Matsuura, Professor at Kyoto Univ., for their continuous encouragement. Finally they wish to acknowledge the thoughtful suggestions of the referees for arising the quality of these three related papers.

\section{REFERENCES}

1) T. Terai, "On calculation of sound fields around three dimensional objects by integral equation methods," J. Sound Vib. 69, 71-100 (1980).

2) Y. Sakurai and Z. Maekawa, "Sound reflection from plane panels and a panel array," J. Acoust. Soc. Jpn. 24, 289-298 (1968) (in Japanese).

3) Y. Ando, "The interference pattern method of measuring the complex reflection coefficient of acoustic materials at oblique incidence," Proc. 6th ICA Tokyo, Paper E-1-9(1968).

4) Z. Kintsl, "Investigation of the sound absorption of wall sections by a pulse technique," Sov. Phys. Acoust. 21, 30-32 (1975).

5) Y. Sakurai and K. Nagata, "Sound reflections of a rigid plane panel and the "live end" composed by those panels," J. Acoust. Soc. Jpn. (E) 2, 5-14 (1981).

6) K. Nagata and Y. Sakurai, "Reflection of a plate with reflection coefficient," Proc. Autumn Meeting of A.S.J., 557-558 (1978) (in Japanese).

7) Y. W. Lee, Statistical Theory of Communication (Univ. of Tokyo Press, Tokyo, 1970), p. 47.

8) K. A. Mulholland, "An impulse method of measuring normal impedance at oblique incidence," J. Sound Vib. 67, 135-149 (1979).

9) T. Nakamura, A. Nakamura, and R. Takeuchi, "Absorption mechanism of porous material for a sound pulse," J. Acoust. Soc. Jpn. 32, 76-82 (1976) (in Japanese).

10) S. I. Thomasson, "Reflection of waves from a point source by an impedance boundary," J. Acoust. Soc. Am. 59, 780-785 (1976).

11) Y. Sakurai, "Sound reflection of a curved rigid panel," J. Acoust. Soc. Jpn. (E) 2, 63-70 (1981).

12) Y. Sakurai, "Sound reflection of plane surface with different reflection coefficients," Proc. Spring Meeting of A.S.J., 471-472 (1979) (in Japanese). 